\title{
Validation of information recorded on general practitioner based computerised data resource in the United Kingdom
}

\author{
Hershel Jick, Susan S Jick, Laura E Derby
}

\begin{abstract}
Objective-To determine the extent of agreement between clinical information recorded on surgery computers of selected general practitioners and similar information in manual records of letters received from hospital consultants and kept in the general practitioners' files.
\end{abstract}

Design-Hospital consultants' letters in the manual records of selected general practitioners were photocopied and the consultants' clinical diagnoses were compared with diagnoses recorded on computer.

Setting-General practices in the United Kingdom using computers provided by VAMP Health for recording clinical information.

Subjects - 2491 patients who received one of three non-steroidal anti-inflammatory drugs and who attended 58 practices whose computer recorded data were considered after a preliminary review to be of satisfactory quality.

Results-Among 1191 patients for whom consultants' letters were forwarded a clinical diagnosis reflecting the diagnosis noted on a consultant letter was present on the computer record for $1038(87 \%)$.

Conclusion-Clinical information available on the computer records of the general practitioners who participated in this study is satisfactory for many clinical studies.

\section{Introduction}

Large computerised data resources have been used successfully for many years in North America to conduct clinical research, particularly in the subject of drug safety. ${ }^{1-4}$ Recently, a large number of general practices in the United Kingdom have placed computers in their surgeries to record medical and administrative information. In November 1989 the Boston Collaborative Drug Surveillance Program started an ongoing retrospective follow up study of a large number of people taking one of three non-steroidal anti-inflammatory drugs to evaluate their relative safety. For this study we used information recorded by general practitioners on surgery computers provided by VAMP Health. The patients were identified directly from the computerised prescriptions recorded on VAMP computers in previous years. To be confident that we had identified and described all important illnesses that developed in the cohorts of users we requested photocopied records (made anonymous) of consultants' letters that were in the original manual records of the patients from the relevant general practitioner.

We used the photocopied letters from the consultants received early in the study to investigate the agreement between the information on diagnosis reported by the consultant and the diagnoses recorded on computer by the general practitioners.

\section{Methods}

VAMP Health is a commercial company that provides surgery computers to general practitioners to allow them to record medical information on their patients. During the past few years interested general practitioners have been offered a "no cost option" plan whereby VAMP Health reimburses the general practitioner monthly for the cost of the computer. About 1000 general practitioners have agreed to participate. Most practices entered the plan between July 1989 and June 1990.

In return for reimbursement general practitioners have agreed to record information in a standard manner, to provide the accumulated information (anonymously) to VAMP, and to the information being used for research purposes. The information that has to be recorded includes demographic information, all medical diagnoses and referrals to hospital, and a record of all prescriptions issued. Prescriptions are produced by the computer, thus ensuring that they are recorded. A special requirement is that the indication for any newly prescribed drug be entered into the computer. The general practitioner may record additional information on immunisations, cervical smear test results, laboratory results, and other relevant medical data. A modification of the OXMIS code is used to denote specific diagnoses, and a coded drug dictionary provided by the Prescription Pricing Authority is used to record drugs. Up to date computerised information is currently collected from each general practitioner on a monthly basis. A few participants have been using the computer for several years. Most started to record information on computer after 1 January 1988.

VAMP provides a 10-12 month period of instruction in using the computer, after which VAMP staff review the accumulated information by using a series of standard criteria to determine whether it seems to be of satisfactory quality. The standard tests currently include a calculation, by practice, of the average number of diagnoses and prescriptions for each patient recorded on computer. These figures are compared with those obtained from other practices and external sources and, where indicated, with previous experience in the practice under review. Results are considered satisfactory if the figures obtained are in reasonable agreement with the average for all practices previously evaluated and thought to be satisfactory. Additional evaluation includes checks to determine whether the indication for newly prescribed drugs is routinely recorded, the outcome of secondary care is recorded, the medical history is consistent with the date of birth, and the outcomes of pregnancies are recorded together with the registration of the offspring. When the results of these preliminary tests were considered by VAMP staff to be satisfactory a practice was categorised as being "up to standard"-that is, satisfactory for research projects. The information from practices considered to be up to standard is reviewed at the time of each subsequent collection of data to ensure that the general practitioners have continued to record the required information. By August 1989 about 75 practices were considered to be up to standard.

Each quarter the Prescription Pricing Authority's information services send every NHS doctor a report compiled from the actual prescriptions dispensed.
Correspondence to: $\mathrm{Dr} \mathrm{H}$

BMY 1991;302:766-8 
This report provides information by practice and by doctor on the number of prescriptions that have been processed by the authority. Abstracts from this report are compared with the computer recorded prescriptions. Greater than $90 \%$ concordance between the authority reports and the VAMP computer figures of recorded prescriptions has been found in practices considered to be up to standard.

A review of the available computer output obtained before January 1990 showed that diagnoses were not always recorded according to a specific OXMIS code; in some cases a reference to the diagnosis (as free text) was placed only in a category available for comments. Similarly, hospital referrals were sometimes noted (as free text) only if they were in a comment category. In the early months of 1990 VAMP introduced new software, which in addition to recording the clinical diagnoses as an OXMIS code also requires that the general practitioner note whether the patient was referred to hospital and, if so, whether it was an inpatient or outpatient referral. It also requires that the general practitioner note whether the diagnosis was a "first ever" diagnosis or one that had been made in the past.

VAMP Health has periodically provided copies of the accumulated computerised data for practices up to standard to our research group, where it is organised into files that are suitable for research. These are similar in structure to files that we have used for many years in studies using the group health cooperative of Puget Sound automated data resource. ' VAMP drug codes, which encompass more than 10000 entries, have been mapped on to a coded drug dictionary, which yields less than 2000 entries, to facilitate research objectives. Both are available for review.

Before using any data resource, particularly one that is based on computer records, it is necessary to determine the quality and completeness of the available information. The research group has, therefore, reviewed the VAMP data for the past two years, during which time we have carried out several studies to evaluate the validity of the critical items of data.

As part of the large follow up study of all computer identified first time users of various non-steroidal antiinflammatory drugs, our research group began in November 1989 to obtain photocopies from the general practitioner manual record of patients' discharge letters received from hospital for a 15 month study period from one month before the first non-steroidal antiinflammatory drug prescription to 14 months after the prescription. Identifying factors for patients and consultants were deleted from all letters to maintain

TABLE I-Newly diagnosed, potentially drug inducible illnesses reported after outpatient consultation according to whether or not there was computer notation of diagnosis

\begin{tabular}{|c|c|c|c|c|c|}
\hline \multirow[b]{2}{*}{ Diagnosis } & \multicolumn{2}{|c|}{ Computer notation } & \multirow[b]{2}{*}{ Diagnosis } & \multicolumn{2}{|c|}{ Computer notation } \\
\hline & Present & Absent & & Present & Absent \\
\hline Cardiovascular disease & 4 & & Myocardial infarction or stroke & 9 & \\
\hline Malignancy & 3 & & Other cardiovascular disease & 10 & \\
\hline Benign tumours & 9 & 1 & Malignancy & 7 & \\
\hline Upper gastrointestinal disease & 9 & & Benign Tumours & 6 & \\
\hline Gall bladder disease & 2 & & Upper gastrointestinal disease & 10 & 1 \\
\hline Lower gastrointestinal disorder & 3 & 1 & Gall bladder disease & 8 & \\
\hline Liver disorder & 4 & & Lower gastrointestinal disorder & 1 & \\
\hline Genitourinary or gynaecological & & & Other gastrointestinal disorder & 8 & 1 \\
\hline disorder & 11 & 1 & Liver disorder & 1 & \\
\hline Skin disorder & 8 & 2 & Genitourinary or gynaecological & & \\
\hline Neuropsychiatric disorder & 12 & 3 & disorder & 8 & 2 \\
\hline Endocrine disorder & 4 & & Skin disorder & 1 & \\
\hline Disease of eye, ear, nose, or throat & 14 & 6 & Neuropsychiatric disorder & 2 & 1 \\
\hline Renal disorder & 1 & & Endocrine disorder & 2 & \\
\hline Haematological disorder & 2 & & Disease of eye, ear, nose, or throat & 3 & 1 \\
\hline Respiratory disorder & 2 & & Renal disorder & 2 & \\
\hline Other & 2 & & Respiratory disorder & 2 & \\
\hline Total & 90 & 14 & Total & 80 & $6^{\star}$ \\
\hline
\end{tabular}

anonymity. Information from the discharge letters was coded by specially trained data abstractors according to an OXMIS code, automated, and then integrated into the original computer files that were generated by the general practitioner.

We reviewed each patient's record manually to determine how often the indication for a new order for a non-steroidal anti-inflammatory study drug had been recorded on computer and to determine the concordance between the information in the computer record and that in the photocopied letters from consultants. Information obtained when a practice was considered to be up to standard was included, and for this study only one consultant's letter for each patient was considered.

For each consultant's letter received we determined whether the recorded illness was diagnosed in the past or newly diagnosed. For newly diagnosed problems we categorised the illness as one which was an indication for the study non-steroidal anti-inflammatory drug (for example, injury, back pain, headache), as one which was not drug inducible (for example, delivery, sterilisation, hernia), or as an illness that was potentially causally connected to a drug. This last category represents those outcomes that are of special interest in a study of illness induced by drugs. In determining whether an illness was potentially drug inducible we used broad inclusion criteria based on past experience with illnesses that have been shown to be drug inducible. For example, we included newly diagnosed benign skin tumours and eye complaints, as in rare instances these have been aetiologically related to drugs, ${ }^{56}$ in addition to conditions such as neoplasms, myocardial infarction and stroke, acute kidney disease, and upper gastrointestinal bleeding, which have more often been associated aetiologically with drugs.

\section{Results}

At the time of our study full information on 2491 patients from 58 practices had been received and reviewed. The indication for first time use of the nonsteroidal anti-inflammatory drug was noted by OXMIS code on the computer record in 2394 (96\%) of patients.

\section{CONSULTANT LETTERS PRESENT}

Among the 2491 patients, photocopied letters from consultants were forwarded to the research group for 1191. Among these 1191 patients a clinical diagnosis reflecting the diagnosis noted in the consultant's letter was present on the computer record in $1038(87 \%)$. The dates present on the computer were closely concordant with the dates in the consultants' letters.

Of the 1191 consultants' letters, 865 noted an outpatient referral and 326 an inpatient referral. Of the 865 outpatient referrals, $104(12 \%)$ were judged to be for a newly diagnosed disease that could be induced by drugs; among the 326 inpatient referrals, $86(26 \%)$ represented newly diagnosed disease that could be induced by drugs.

We further evaluated the 190 patients with newly diagnosed potentially drug inducible illness to determine the specific illnesses that were present and the concordance between the clinical and computer records. Among the 104 patients with outpatient referrals the diagnosis was present on the computer file in $90(87 \%)$. The diagnoses (by category) made in patients with outpatient referrals are given in table I according to whether or not the diagnosis was on the computer record. Diagnoses related to cardiovascular, gastrointestinal, and endocrine disorders as well as those of malignancy tended to be routinely recorded on the computer.

The diagnosis was on the computer file for $80(93 \%)$ of the 86 patients with inpatient referrals. Table II 
shows details of the diagnoses in the 86 patients according to whether or not the diagnosis was on the computer file. All cases of cardiovascular, malignant, liver, renal, endocrine, and skin disorders and all but one case of well defined gastrointestinal illness were recorded on the computer.

As noted above, diagnoses were recorded on the computer for 170 patients with newly diagnosed drug inducible disease. The diagnosis was recorded with an appropriate OXMIS code in 163 (96\%). In the remaining seven patients the diagnosis was mentioned (uncoded) only in the comments category.

\section{CONSULTANT LETTER NOT PRESENT}

Among the 2491 patients there were 1300 (55\%) for whom no consultant's letter was present in the clinical record. A review of the computer record provided no evidence that a referral to hospital had occurred in 1272 of these. There was a newly diagnosed drug inducible illness noted on the computer in only four of the remaining 28 patients. The diagnoses in these four patients were acute myocardial infarction, stroke, skin lesion, and eye disorder. It may be noted that the patients with myocardial infarction and stroke as a computer recorded diagnosis may have been cared for at home, in which case a hospital consultant's letter would not have existed. In the remaining two patients a consultant's letter would not have been present if the patients had not kept their appointments.

\section{Discussion}

The results of our study show that the information available on the VAMP computers used by general practitioners in the United Kingdom is reasonably complete with regard to drugs prescribed and clinical illnesses that are diagnosed by the general practitioner or a consultant physician.

Aetiological studies of drug safety generally entail identifying people who did or did not receive particular drugs and those who subsequently developed an illness that might have been caused by the drugs under study. Our study shows that over $90 \%$ of all drug prescriptions given to patients were recorded on the computer and subsequently dispensed. If this is true, a study based on the VAMP data would involve minimal misclassification of drug exposure. In addition, the results of this study show that the indication for newly prescribed drugs was recorded in over $95 \%$ of cases. As most illnesses are treated with a drug the presence of the indication for drug use in itself provides for a list of illnesses that have been diagnosed. Illnesses that are not treated with a drug or occur in people who are not referred may not be recorded on computer. Such illnesses, however, are rarely of interest in epidemiological studies.

There is always some uncertainty about whether a patient actually fills a prescription and whether the patient, after filling a prescription, actually takes the drug prescribed. This is a particular concern for drugs prescribed for short term use. Studies based on prescription records presume that most people who receive a prescription for a drug actually take the drug and that few, if any, people who do not receive a prescription for that drug actually take it. The degree of misclassification of exposure in a formal study depends on the particular drug(s) under study. For example, in a study of non-steroidal anti-inflammatory drugs these drugs are almost always prescribed for pain, and the assumption that most patients who receive a prescription will actually take the drug seems justified. In a study of the effects of drugs such as insulin, oral contraceptives, and replacement oestrogens it seems reasonable to presuppose that little misclassification of exposure is present.

There was a high concordance between the consultants' letters provided by the general practitioners and the information recorded on the computer. This was particularly true for patients admitted to hospital for illnesses that are often of particular concern in large scale studies of drug safety. In the current series $87 \%$ of all consultants' letters were associated with a concordant diagnosis on the computer. Moreover, more than $90 \%$ of the newly diagnosed drug inducible illnesses that lead to admission to hospital were recorded on the computer. This degree of quality and completeness means that misclassification of diagnoses would be minimal in a study based on the VAMP data resources.

The Boston Collaborative Drug Surveillance Program has completed a study comparing the relative frequency of clinically diagnosed hypoglycaemia in patients taking animal and human insulin based on the VAMP resource, ${ }^{7}$ and we are currently carrying out additional studies of drug safety with this resource. Our experience in obtaining the relevant data required to properly complete these studies shows us that this resource is adequate to accomplish many valid studies of drug safety.

A critical requirement for the proper conduct of studies of drug safety is the availability of original clinical records to evaluate the evidence for a clinical diagnosis that has been assigned to a patient. The general practitioners that we have been working with have been extremely cooperative in providing records where required for our studies.

By April 1990 the information available to us from general practitioners using VAMP computers encompassed more than 1.5 million people who have had clinical information recorded on computer for an average of about two years. There are currently more than 50 drugs for which cohorts of more than 20000 users can be identified. We expect that in the near future as more practices have completed their training period the population size will increase to at least 3 million. We conclude that the large size, together with the high quality and completeness of the information that we have reported to be present on selected VAMP computers, provides a unique resource for a wide range of clinical studies that can be accomplished quickly and at reasonable cost.

We thank the staff at VAMP and the participating general practitioners for their excellent cooperation. This research was supported by a grant from Ciba-Geigy Pharmaceuticals. The Boston Collaborative Drug Surveillance Program is supported partly by the Food and Drug Administration (cooperative agreement FD-U-000071-09) and by grants from: Boots Pharmaceuticals, Burroughs Wellcome, CibaGeigy Corporation, Hoffmann-La Roche, McNeil Consumer Products, Pfizer, and SmithKline Beecham Pharmaceuticals.

$$
\begin{aligned}
& 1 \text { Jick H, Madsen S, Nudelman PM, et al. Postmarketing follow up at group } \\
& \text { health cooperative of Puget Sound. Pharmacotherapy 1984;4:99-100. } \\
& 2 \text { Ray WA, Griffin MR, Schaffner W, Baugh DK, Melton LJ. Psychotropic drug } \\
& \text { use and the risk of hip fracture. N Engl f Med 1987;316:363-9. } \\
& 3 \text { West R. Saskatchewan health data bases: a developing resource in progress in } \\
& \text { pharmacoepidemiology. American fournal of Preventive Medicine } 1988 \\
& \text { (suppl):25-7. } \\
& 4 \text { Tilson HH. A proactive approach to monitoring for adverse drug reactions. } \\
& \text { fournal of Clinical Research and Drug Development 1989;3:39-51. } \\
& 5 \text { Findlay GH, Hull PR. Eruptive tumours on sun-exposed skin after benoxa- } \\
& \text { profen. Lancet 1982;ii: } 95 \text {. } \\
& 6 \text { Skegg DCG, Doll R. Frequency of eye complaints and rashes among patients } \\
& \text { receiving practolol and propanolol. Lancet 1977; ii:475-8. } \\
& 7 \text { Jick H, Hall GC, Dean AD, Jick SS, Derby LE. A comparison of the risk of } \\
& \text { hypoglycemia between users of human and animal insulins. I. Experience in }
\end{aligned}
$$
the United Kingdom. Pharmacotherapy 1990;10:395-7.

(Accepted 1 February 1991) 